
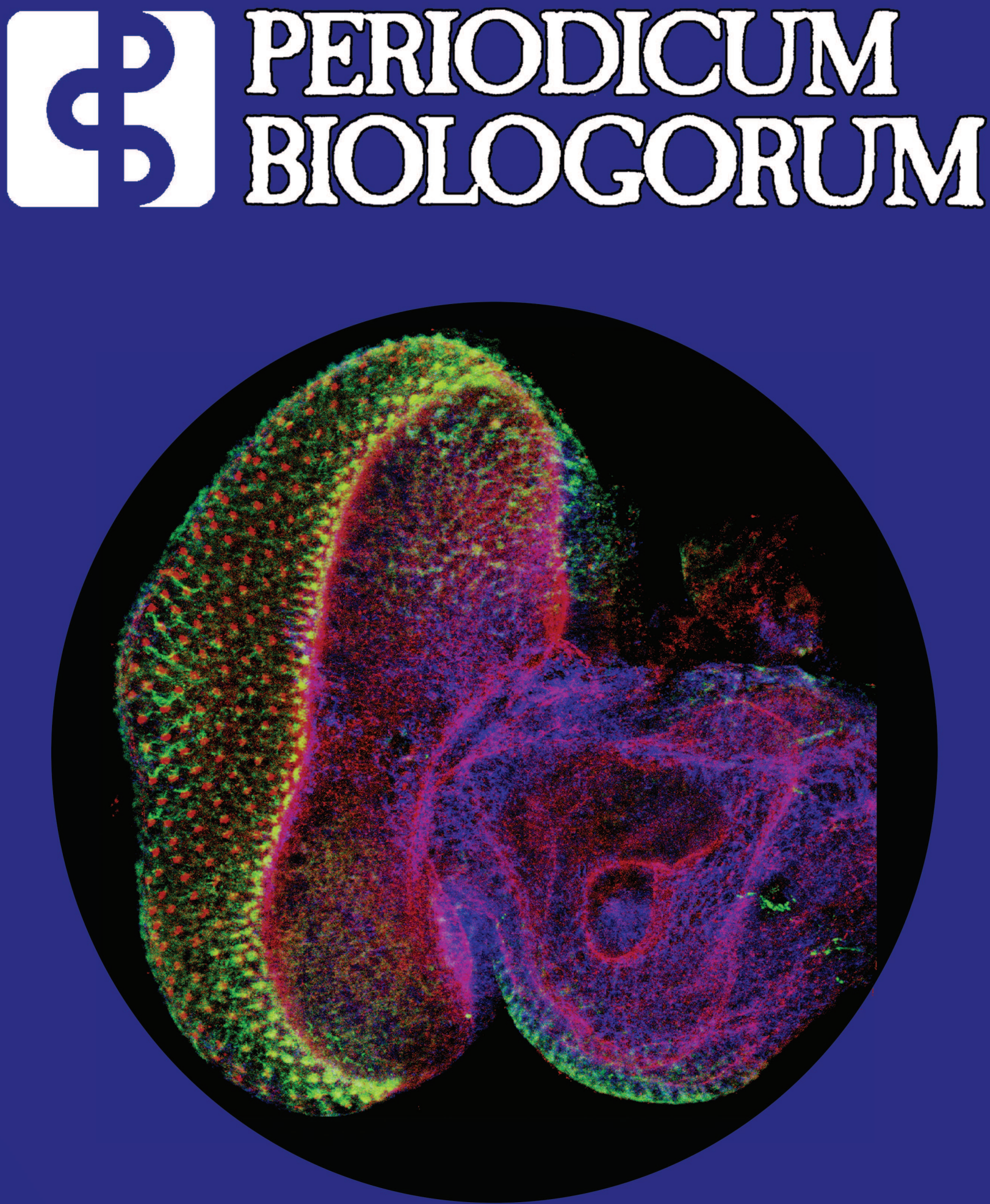
DOI: 10.18054/pb.v119i1.bb1

\section{BEAUTY OF BIOLOGY}

We have decided, from time to time, to put on cover image(s) that are not necessarily related to one of the papers published in that issue. These images will be related to beauty of biology, and will be accompanied with few basic data about that image (the author, what does it present, where and how it was made). Hope you will enjoy it.

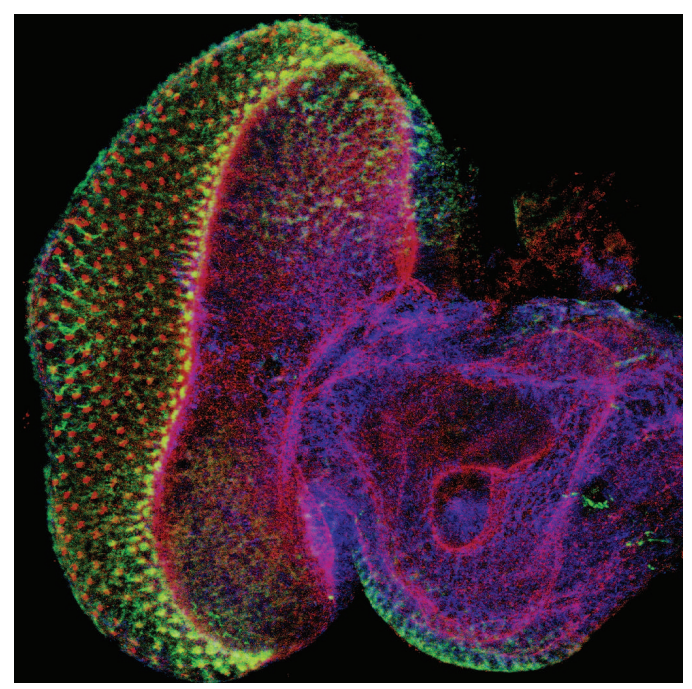

About the image:

„The rudiments for the formation of a whole body architecture in holometabolous insects are already present during larval development, before metamorphosis occurs. In this picture we can observe the eye imaginal disc of a Drosophila melanogaster larva; a big portion of the tissue we see (blue) will serve as support, separated by a furrow of another part (left side) that will be specified to produce the ommatidia, the units that form the compound eye typical of this group. Only one of each 4 nervous cells will be selected (red dots) to send axons (green) to the brain: the connection between the eye and the brain is essential to produce a functional vision and is stablished during this developmental stage.

To study these processes, we employ transgenic flies expressing GFP together with immunohistochemistry techniques. Imaging was performed using a Leica upright DM-SL confocal microscope and Z-scan was assembled and adjusted with ImageJ. This picture was made by Dr. Raquel Perez-Gomez in Instituto de Neurociencias de Alicante (CSIC/UMH), Spain.“ 